TENDENCIAS

Revista de la Facultad de Ciencias

Económicas y Administrativas.

Universidad de Nariño

ISSN 0124-8693 ISSN-E 2539-0554

Vol. XIX No. 2 - 2० Semestre 2018,

Julio - Diciembre - Páginas 74-91

\title{
COMPORTAMIENTO ASIMÉTRICO DE LOS COSTOS EN EL SECTOR DE LA CONSTRUCCIÓN DEL ECUADOR
}

\author{
ASYMMETRIC BEHAVIOR OF COSTS IN THE CONSTRUCTION \\ SECTOR OF ECUADOR
}

\section{COMPORTAMENTO ASSIMÉTRICO DOS CUSTOS NO SECTOR DA CONSTRUÇÃO DO EQUADOR}

\section{CORDOVA LEÓN_José Fernando, DUQUE ESPINOZA_Gabriela Monserrath, ÁLVAREZ ARGÜELLO_Carolina Denisse}

Doctor en Ciencias Económicas con mención en Ciencias Empresariales, Universidad Nacional de Córdoba, UNC. Docente Facultad de Ciencias de la Administración, Universidad del Azuay. Email: jfcordova@uazuay.edu.ec, Ecuador.

Magister en contabilidad y finanzas con mención en gerencia y planeamiento tributario, Universidad del Azuay, UDA. Docente Facultad de Ciencias de la Administración, Universidad del Azuay. Email: gduque@uazuay.edu.ec, Ecuador.

Ingeniera en Contabilidad y Auditoría, Universidad del Azuay, UDA. Email: denisse. alvarez91@gmail.com, Ecuador.

Recibido: 30 de septiembre de 2018 Aprobación definitiva: 6 de diciembre de 2018

\section{DOI: http://dx.doi.org/10.22267/rtend.181902.98}

\section{RESUMEN}

El sector de la construcción en el Ecuador genera gran interés por su aporte al desarrollo económico y territorial del país. Las metodologías y herramientas de análisis para este sector de actividad económica resultan insuficientes, por tanto, es necesario evaluar nuevos mecanismos para una gestión y control efectivo por parte de los gerentes inmobiliarios. El presente trabajo tiene por objeto analizar el comportamiento de los costos en el sector de la construcción, con la finalidad de evaluar el nivel de asimetría de los costos y gastos en relación al cambio en 
el nivel de ventas. Los datos fueron analizados mediante estadística descriptiva y modelos de regresión, considerando una muestra de 381 firmas durante cuatro años consecutivos. El estudio confirma la teoría de Anderson et al. (2003) la cual sostiene que los costos aumentan en mayor proporción cuando las ventas aumentan, $\mathrm{y}$ disminuyen en menor proporción cuando las ventas decrecen, concepto que orientó el análisis de la presente investigación.

Palabras Clave: Asimetría de costos, costos pegadizos, costos de operación. JEL: M20, M210, M110

\begin{abstract}
The construction sector in Ecuador generates great interest for its contribution to the economic and territorial development of the country. The methodologies and analysis tools for this sector of economic activity are insufficient, therefore, it is necessary to evaluate new mechanisms for effective management and control by real estate managers. The purpose of this paper is to analyze the behavior of costs in the construction sector, in order to assess the level of asymmetry of costs and expenses in relation to the change in the level of sales. The data were analyzed using descriptive statistics and regression models, considering a sample of 381 signatures during four consecutive years. The study confirms the theory of Anderson et al. (2003) which argues that costs increase in greater proportion when sales increase, and decrease in smaller proportion when sales decrease, concept that guided the analysis of the present investigation.
\end{abstract}

Keywords: Cost asymmetry, catchy costs, operating costs.

JEL: M20, M210, M110

\title{
RESUMO
}

O setor de construção no Equador gera grande interesse por sua contribuição ao desenvolvimento econômico e territorial do país. As metodologias e ferramentas de análise para este setor de atividade econômica são insuficientes, portanto, é necessário avaliar novos mecanismos de gestão e controle efetivos por gestores imobiliários. O objetivo deste trabalho é analisar o comportamento dos custos no setor de construção, a fim de avaliar o nível de assimetria de custos e despesas em relação à mudança no nível de vendas. Os dados foram analisados por meio de estatística descritiva e modelos de regressão, considerando uma amostra de 381 assinaturas durante quatro anos consecutivos. O estudo confirma a teoria 
de Anderson et al. (2003), que argumenta que os custos aumentam em maior proporção quando as vendas aumentam, e diminuem em menor proporção quando as vendas diminuem, conceito que norteou a análise da presente investigação.

Palavras-chave: Assimetria de custo, custos atrativos, custos operacionais. JEL: M20, M210, M110

\section{INTRODUCCIÓN}

El ambiente empresarial cambiante y dinámico en el cual las organizaciones se desarrollan, requiere que estas implementen estrategias de constante crecimiento y de desempeño sostenido, que les permita aprovechar oportunidades y hacer frente a amenazas que se presentan en sus diferentes campos de acción.

Lo gerentes y encargados de la toma de decisiones empresariales requieren de información que les permita optimizar sus recursos, ser competitivos y maximizar la rentabilidad, consiguiendo generar valor para sus accionistas.

La contabilidad de costos, como parte de la contabilidad financiera, se encarga de medir y cuantificar los recursos utilizados para producir un bien o prestar un servicio, sin brindar información sobre el comportamiento o las causas que los producen. Frente a estas necesidades, la gestión de costos brinda herramientas adicionales que permiten plantear acciones estratégicas buscando optimizar costos y administrar de mejor manera los recursos, con el fin de mejorar los resultados de una organización; pero, algunas de sus herramientas mantienen el concepto tradicional de que el costo está en función de los volúmenes de producción, y por ende de sus ventas.

Los cambios en los niveles de ingresos de una organización obligan a los gerentes a evaluar de manera inmediata las repercusiones en su rentabilidad, y a realizar los ajustes necesarios en sus costos y gastos, teniendo como riesgo constante la recuperación o no de los niveles esperados de ventas. La gerencia deberá evaluar el costo que representa prescindir de ciertos recursos, comparado con los montos por mantener recursos improductivos esperando se alcancen los ingresos deseados. Por la importancia que tiene el manejo de los costos y gastos en el desempeño empresarial, es necesaria información constante que permita 
analizar el comportamiento de estas variables, que evaluadas junto con otras herramientas financieras permitan el planteamiento de acciones y políticas para el manejo económico de las organizaciones.

Es así, que el análisis de los costos pegados (sticky costs) presenta una metodología adicional para evaluar el comportamiento de los costos y gastos, sosteniendo que el ajuste en estas variables se retrasa más cuando el volumen de ventas disminuye que cuando aumenta, presentando asimetría en la relación de los ingresos con los costos y gastos, a diferentes niveles de actividad. Esta herramienta nace por deficiencias presentadas en los modelos de costeo existente, y por la poca precisión que presentan para la toma de decisiones.

El análisis de Cooper y Kaplan (1988) sugiere que los gerentes de las empresas tienden a modificar los costos cuando las ventas aumentan, pero no hacen lo mismo cuando las ventas disminuyen, aporte que dio inicio a investigaciones sobre el comportamiento asimétrico de los costos.

Posterior a esto, Anderson, Banker, y Janakiraman (2003) son los primeros en definir el término "sticky costs", refiriéndose a los costos que aumentan en mayor proporción cuando las ventas aumentan en un porcentaje, que lo que disminuyen cuando éstas decaen en la misma proporción.

Es importante entonces, generar análisis sobre la asimetría que presentan los costos en los diferentes sectores de actividad, y en particular en la construcción, considerando que la información que presenta será de vital importancia para una eficiente toma de decisiones, permitirá realizar planificación a largo plazo, y minimizar los riesgos; conociendo que si bien la estructura de costos de una firma, la situación del mercado en el que se desarrolla y las expectativas económicas son importantes, el desempeño organizacional está medido por el impacto en el rendimiento empresarial, y este depende de la velocidad con que la gerencia tome decisiones acertadas ante cambios en la actividad, que se reflejen en los resultados a corto, mediano y largo plazo.

La presente investigación tiene por objeto analizar el comportamiento de los costos y gastos en el sector de la construcción, con el fin de evaluar el nivel de asimetría de estas variables en relación con el cambio en el nivel de actividad. 
El estudio se enfoca en 4418 sociedades registradas en la Superintendencia de Compañías, Valores y Seguros que se dedican a la actividad de la construcción en el Ecuador, entre los años 2012 al 2015.

Se plantea como hipótesis a contrastar:

H1. Las empresas ecuatorianas del sector de la construcción muestran un comportamiento asimétrico en el total de costos y gastos, como en los gastos de personal, frente a los cambios en los niveles de ventas.

\section{FUNDAMENTOS TEÓRICOS}

La medición y estudio de los costos y gastos aporta con información relevante que apoya la toma de decisiones sobre actividades, operaciones de negocio y áreas funcionales, para la planeación de los recursos empresariales dentro del marco de referencia de la organización. Permite formular, comunicar e implementar estrategias encaminadas al logro de un desempeño financiero, social y ambiental sostenible.

Según Horngren (2012) el nivel de actividad de una organización es definida como un generador de costos cuando hay una relación de causa y efecto entre un cambio en el nivel de actividad o volumen y un cambio en el nivel de costos totales, por lo que analizar este comportamiento brindará información que conduzca a ajustar las capacidades empresariales con las oportunidades de mercado para cumplir con los objetivos organizacionales. Además, apoya al correcto manejo y optimización de recursos, permitiendo ser competitivos y generar valor para el cliente, buscando maximizar el rendimiento.

El análisis de Cooper y Kaplan (1988) sugiere que es necesario considerar primero el comportamiento de los costos en lugar del nivel de actividad, ya que los costos y gastos están en función de las decisiones a corto y medio plazo que toma la administración, y lo segundo depende de la demanda del mercado. Malcom (1991) inicia el desarrollo del tema sobre costos asimétricos mediante un estudio del manejo de materiales en una empresa, en la cual al disminuir las ventas no se realizan despidos de personal de manera inmediata, en espera a la reacción de la demanda del mercado, ya que al firmarse contratos de personal a largo plazo no pueden ser terminados por las erogaciones que implicaría, concluyendo que no todos los costos tienen relación con el volumen de las ventas. 
Anderson, Banker y Janakiraman (2003) estudiaron la relación entre los costos y las ventas mediante un modelo de regresión lineal logarítmica utilizando variables como: el producto interno bruto, la proporción del activo total sobre los ingresos y la cantidad de personal sobre los ingresos. Determinan la pegajosidad de los costos en 7629 empresas, encontrando que los costos son menos pegajosos cuando la empresa tiene menores ingresos en períodos sucesivos, y más pegajosos cuando el producto interno bruto crece, y cuando las empresas tienen mayores activos y mano de obra. Concluyen que los costos aumentan en mayor proporción cuando las ventas aumentan en un porcentaje, que lo que disminuyen cuando éstas decaen en la misma proporción, atribuyendo a la administración el comportamiento de los costos debido a la toma de decisiones empresariales.

Algunos autores demuestran la importancia del tiempo para la pegajosidad de los costos. Banker, Byzalov y Plehndujowich (2014) realizan su estudio en el sector manufacturero con una muestra de 60.000 empresas en donde se determina que los costos son más pegadizos en períodos de tiempo más cortos ante una mayor incertidumbre de la demanda; al igual que el estudio de Ribeiro de Medeiros y De Souza Costa (2004) quienes indican que en períodos más largos de análisis los costos se vuelven menos pegajosos. Por el contrario, Cunha, Silva y Correia (2014) analizaron con el método de regresión lineal logarítmica de estimados por mínimos cuadrados la pegajosidad de los costos en relación a los ingresos de 669 empresas de Latinoamérica, concluyendo que los costos son más pegadizos en períodos más largos debido probablemente a las leyes laborales de los países de estudio.

Otros autores relacionan la pegajosidad de los costos con variables como el clima económico, nivel de control, tamaño de las empresas, entre otros. Nassirzadeh, Saei, Salehi y Bayegi (2013) estudian las compañías cotizadas en la bolsa de Teherán en un período de 10 años, en 21 industrias diferentes, concluyendo que los gastos de ventas son menos pegajosos cuando existen menores ventas en períodos anteriores. West (2003) al investigar los costos concluye que los costos variables, en su mayoría, tienden a ser más pegadizos cuando no se tiene el debido control de los mismos. Porporato y Werbin (2010) fundamenta su estudio en una muestra de los costos en los bancos de Argentina, Brasil y Canadá atribuyendo el comportamiento de los costos al clima económico como los activos, costos fijos e incertidumbre del mercado. Stimolo (2016) en 
un estudio analiza la pegajosidad de los costos a corto plazo en las empresas argentinas que cotizan acciones en el periodo 2004 - 2012, las conclusiones muestran un comportamiento pegajoso en la mayoría de los casos. Cagigal y Petrovic (2017) analizan en empresas ecuatorianas la relación entre los costos de ventas y las ventas donde se mantiene la simetría de los costos, sin embargo, con respecto a los gastos de ventas, sí se genera una asimetría resaltando que el tamaño de las empresas y la presentación contable infieren en su comportamiento.

Las investigaciones de diversos autores Banker et al. (2014), Ribeiro de Medeiros et al. (2004), Cunha, et al. (2014), Nassirzadeh et al. (2013), Porporato y Werbin (2010), Stimolo (2016), Cagigal y Petrovic (2017), demuestran que la asimetría y pegajosidad de los costos se cumple, independiente del lugar, periodo de tiempo y actividad económica que desarrollen las organizaciones sujetas a estudio.

Anderson et al. (2013), luego de su estudio en el cual determina que por un incremento del $1 \%$ en ventas los costos aumentan en $0,55 \%$, y que al disminuir en el mismo porcentaje los costos se reducen únicamente en $0,35 \%$, indica que los costos se consideran pegados cuando la magnitud del aumento en los costos en relación al aumento en el volumen de ventas es mayor que la magnitud de la disminución en los costos por una disminución equivalente en la actividad. Los costos pegadizos se definen como una relación asimétrica entre los costos (administrativos, generales de ventas) y el volumen de actividad de una empresa, desvirtuando el concepto tradicional de que los costos son simétricos en proporción a las ventas (Anderson, 2013).

Las decisiones empresariales inciden directamente en la pegajosidad de los costos, pues al enfrentar una disminución de actividad, la gerencia debe decidir qué costos mantener o ajustar, considerando factores internos y externos a la organización. Los recursos que están comprometidos mediante contratos suelen tener mayor dificultad para ser ajustados, debido a que los costos por liquidar estos acuerdos son altos, teniendo en ocasiones que mantener recursos innecesarios ante una disminución de ventas, en lugar de realizar cambios que permitan minimizar costos y recontratar estos recursos posteriormente. Además, aspectos como los activos, empleados y capital de trabajo marcan la diferencia en el nivel de actividad lo que influirá de manera positiva o negativa en el comportamiento de los costos. 
La importancia de analizar el comportamiento de los costos en relación a las ventas para demostrar la asimetría da lugar al desarrollo de un modelo conocido como ABJ, propuesto por Anderson, Banker y Janakiraman fundamentado principalmente en la función de costos de Cobb-Douglas. Anderson et al. (2003) utilizan tres diferentes modelos para desarrollar sus hipótesis, a través del método de mínimos cuadrados ordinarios, los cuales le permitieron demostrar las hipótesis de los estudios realizados.

El modelo $\mathrm{ABJ}$ es un modelo de regresión, que permite evaluar la relación entre los cambios en los costos cuando los ingresos aumentan o disminuyen en dos períodos sucesivos con el fin de evidenciar el comportamiento de estas variables, brindando información que apoye a la eficiente toma de decisiones gerenciales.

\section{METODOLOGÍA}

La población comprendió 4418 empresas de la construcción (corte año 2015) registradas en la Superintendencia de Compañías, Valores y Seguros bajo el nivel F41 ${ }^{1}$ : Construcción de edificios, en el Ecuador; compañías que fueron analizadas durante un periodo de cuatro años, a partir del 2012. La muestra fue probabilística, con un nivel de confianza del 0,95. Si bien se determinó una muestra de 354 observaciones, fue posible obtener datos de 381 empresas constructoras para cada uno de los años.

Para el estudio se consideraron tres diferentes variables: los ingresos por ventas $^{2}$, que incluye todas las entradas de dinero que se han generado en la empresa producto del giro del negocio, variable incluida solamente para empresas que registran variación en su nivel de ingresos; el total de costos y gastos los cuales son los valores en que ha incurrido la empresa para llevar a cabo su actividad económica; y finalmente, el gasto en personal tomado del estado de resultados, que incluye los costos de mano de obra y de personal administrativo.

1 La Superintendencia de Compañías, Valores y Seguros del Ecuador, lleva en su página web oficial el Directorio de Compañías, clasificándolas por actividad económica, según CIIU4 (Clasificación Industrial Internacional Uniforme). http://appscvs.supercias.gob.ec/portalInformacion/ sector_societario.zul

2 Los ingresos son aumentos en los activos, o disminuciones en los pasivos, que resultan en aumentos en el patrimonio, distintos de los relacionados con las contribuciones de los tenedores de reclamaciones del patrimonio. (Marco conceptual NIIF, 2018) 
Previo al contraste de variables, y por motivos de inflación, fue necesario el ajuste de los ingresos, costos y gastos totales, y gastos de personal de los años 2012, 2013 y 2014, de manera que los datos sean medibles y comparables. Ver tabla $\mathrm{N}^{\mathrm{o}} 1$

\section{Tabla 1}

Inflación anual en Ecuador 2012- 2014

\begin{tabular}{|c|c|}
\hline AÑO & INFLACIÓN \\
\hline 2012 & 0,0416 \\
\hline 2013 & 0,027 \\
\hline 2014 & 0,0367 \\
\hline
\end{tabular}

Fuente: Instituto Nacional de Estadística y Censos INEC años 2012 al 2015

Los valores atípicos fueron filtrados mediante el criterio de Chauvenet ${ }^{3}$, el cual, tomando la media como punto de equilibrio entre los valores, permite controlar la dispersión de los datos.

Para el contraste de las hipótesis, se realizó en primera instancia un análisis estadístico que permitió obtener elasticidades de los costos en relación con las ventas; y en segunda instancia, se aplicó el modelo de regresión propuesto por Anderson, Banker y Janakiraman, el cual determinó el nivel de asimetría de los costos ante los diferentes cambios de actividad empresarial.

\section{RESULTADOS Y DISCUSIÓN}

Como estadística inicial se analizaron los datos en conjunto, registrando en la variable ventas un total de 1138 observaciones, en el total de costos y gastos 1186 datos, y finalmente los gastos de personal con 1078 valores.

\section{Tabla 2}

Datos estadísticos de las variables ventas, costos y gastos, y gastos de personal 2012-2015

3 El criterio de Chauvenet es un método cuantitativo que elimina datos atípicos en los que no se puede suponer la distribución normal; se basa en la magnitud que posee un valor dudoso respecto de la media de los demás. 


\begin{tabular}{|c|c|c|c|c|c|c|}
\hline Variable & Media & $\begin{array}{c}\text { Desviación } \\
\text { estándar }\end{array}$ & Percentil 25 & Percentil 50 & Percentil 75 & $\begin{array}{c}\text { Coeficiente de } \\
\text { variación }\end{array}$ \\
\hline $\begin{array}{c}\text { Ventas } \\
\text { netas }\end{array}$ & $\$ 1.431 .745,30$ & 3577986,81 & $\$ 95.380,79$ & $\$ 395.508,70$ & $\$ 1.239 .718,18$ & 2,50 \\
\hline $\begin{array}{c}\text { Total de } \\
\text { costos } \\
\text { yastos }\end{array}$ & $\$ 1.296 .043,39$ & 3013429,39 & $\$ 5.615,51$ & $\$ 160.982,01$ & $\$ 761.745,98$ & 2,63 \\
\hline $\begin{array}{c}\text { Gastos de } \\
\text { personal }\end{array}$ & $\$ 290.599,84$ & 748865,47 & $\$ 28.461,26$ & $\$ 84.083,72$ & $\$ 230.901,53$ & 2,58 \\
\hline
\end{tabular}

Fuente: Elaboración propia.

La tabla $\mathrm{N}^{\mathrm{o}} 2$ muestra el resumen estadístico, que permitió comparar la media con la mediana de cada variable (percentil 50). Se observa que en todos los casos la dispersión es alta y la media se encuentra sobre el percentil 75, en concordancia con el estudio de Stimolo (2013) y Cagigal y Petrovic (2017) en el cual refieren que el comportamiento es asimétrico hacia la derecha de la distribución.

Para el posterior contraste de resultados, la variable Ventas fue segmentada y analizada en dos grupos:

- Empresas que aumentaron sus ventas, con sus porcentajes de variación.

- Empresas que disminuyeron sus ventas, con sus porcentajes de variación.

En base a esta segmentación, se determinó para cada grupo el cambio en las tasas de costos y gastos, y gasto en personal, con sus respectivos comparativos en los años de estudio: 2012-2013, 2013-2014 y 2014-2015.

En la tabla $\mathrm{N}^{\mathrm{o}} 3$ se presenta la información consolidada para el periodo de análisis, exponiendo las tasas de variación de ventas, costos y gastos y gastos de personal a manera de resumen, con sus medias, desviación estándar, percentiles y coeficientes de variación.

\section{Tabla 3}

Estadísticos básicos para tasas de variación de ventas, costos y gastos y gastos de personal, años 2012-2015 
Comportamiento asimétrico de los costos en el sector de la construcción del Ecuador Cordova León_José Fernando, Duque Espinoza_Gabriela Monserrath, Álvarez Argüello_Carolina Denisse

\begin{tabular}{|c|c|c|c|c|c|c|c|}
\hline Variables & Media & $\begin{array}{c}\text { Desviación } \\
\text { Estándar }\end{array}$ & $\begin{array}{c}\text { Percentil } \\
\mathbf{2 5}\end{array}$ & Percentil 50 & $\begin{array}{c}\text { Percentil } \\
\mathbf{7 5}\end{array}$ & $\begin{array}{c}\text { Coeficiente de } \\
\text { Variación }\end{array}$ & N \\
\hline \multicolumn{7}{|c|}{ En períodos de disminución anual de ventas } \\
\hline Ventas & 0,42 & 0,29 & 0,17 & 0,39 & 0,64 & 0,68 & 374 \\
\hline $\begin{array}{c}\text { Costos y } \\
\text { gastos }\end{array}$ & 0,12 & 1,19 & 0,11 & 0,32 & 0,66 & 9,62 & 362 \\
\hline $\begin{array}{c}\text { Gastos de } \\
\text { personal }\end{array}$ & 0,09 & 0,57 & $-0,07$ & 0,12 & 0,43 & 6,68 & 325 \\
\hline \multicolumn{7}{|c|}{ En períodos de aumento anual de ventas } & \\
\hline Ventas & 1,44 & 2,21 & 0,17 & 0,54 & 1,58 & 1,54 & 353 \\
\hline $\begin{array}{c}\text { Costos y } \\
\text { gastos }\end{array}$ & 1,35 & 2,18 & 0,10 & 0,59 & 1,78 & 1,62 & 327 \\
\hline $\begin{array}{c}\text { Gastos de } \\
\text { personal }\end{array}$ & 0,90 & 2,55 & $-0,07$ & 0,24 & 0,68 & 2,85 & 337 \\
\hline
\end{tabular}

Fuente: Elaboración propia.

\section{Análisis descriptivo de elasticidades}

Para la obtención del coeficiente de elasticidad, fue necesario calcular el cociente entre tasas de variación de costos y gastos, y la tasa de variación de ventas. En la tabla $\mathrm{N}^{\mathrm{0}} 4$, se presentan las elasticidades resultantes, evidenciando la existencia de asimetría ante los cambios en el nivel de actividad.

\section{Tabla 4}

Elasticidades en el sector de la construcción de los periodos 2012-2015 en el Ecuador

\begin{tabular}{|c|c|c|c|c|}
\hline Variables & Variación & $\%$ de variación & Elasticidad & n \\
\hline \multicolumn{5}{|c|}{ En períodos de disminución anual de ventas } \\
\hline Ventas & 0,42 & 41,86 & $1 \%$ & 374 \\
\hline Costo de ventas y gastos & 0,12 & 12,40 & $0,30 \%$ & 362 \\
\hline Gastos de personal & 0,09 & 9 & $0,20 \%$ & 325 \\
\hline \multicolumn{7}{|c|}{ En períodos de aumento anual de ventas } \\
\hline
\end{tabular}




\begin{tabular}{|c|c|c|c|c|}
\hline Ventas & 1,44 & 143,52 & $1 \%$ & 353 \\
\hline Costo de ventas y gastos & 1,35 & 134,66 & $0,94 \%$ & 327 \\
\hline Gastos de personal & 0,90 & 89,55 & $0,62 \%$ & 337 \\
\hline
\end{tabular}

Fuente: Elaboración propia.

La Tabla $N^{\circ} 4$ muestra que ante un incremento de las ventas en 1\%, los costos y gastos aumentan en 0,94\%, mientras que en los años en los que hubo disminución de ventas, los costos solamente se redujeron en un $0,30 \%$. De la misma manera con los gastos de personal, ante un incremento del 1\% en las ventas, los gastos de personal aumentan $0,62 \%$, mientras que, ante la disminución de las ventas en el mismo porcentaje, los costos y gastos de personal tan sólo disminuyen en $0,20 \%$. Al realizar este análisis se pudo observar claramente que los costos no tienen el mismo comportamiento conforme las ventas aumentan o disminuyen, por lo tanto, se puede presumir que éstos se mantienen pegados y por lo tanto son asimétricos. La siguiente figura resume este comportamiento:

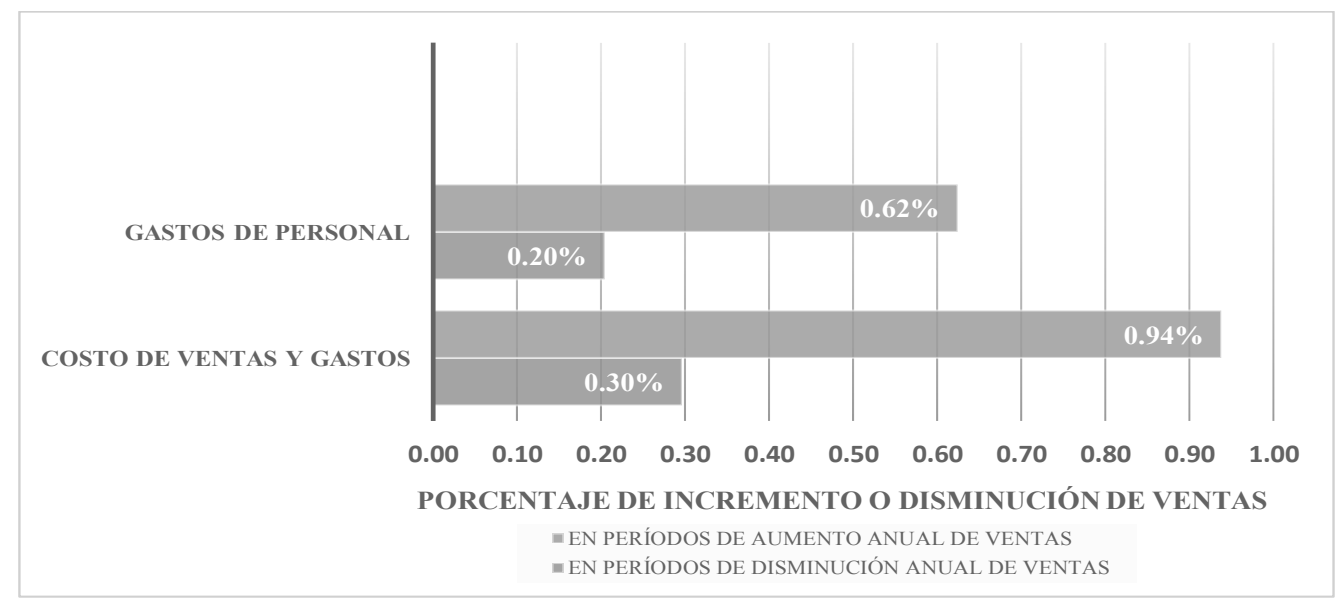

\section{Figura 1}

Comportamiento asimétrico de los costos y gastos en el sector de la construcción ecuatoriana años 2012-2015

Fuente: Elaboración propia.

\section{Modelo lineal}

La regresión lineal permite un acercamiento inicial al nivel de correlación de variables. El modelo relacionó las ventas con el total de costos y gastos, así como las ventas con los gastos de personal, en donde $\beta_{0}$ representó la constante, 
y $\beta_{1}$ la pendiente o velocidad en la que se incrementaron los costos y gastos, y los gastos de personal en proporción a las ventas. $U_{i}$ se consideró el valor aleatorio de los errores.

$$
\begin{gathered}
\text { Costos y gastos de ventas }=\beta_{0}+\beta_{1} \text { Ventas }+U_{i} \\
\text { Gastos de Personal }=\beta_{0}+\beta_{1} \text { Ventas }+U_{i}
\end{gathered}
$$

\section{Tabla 5}

Correlación de variables y factor de ajuste

\begin{tabular}{|c|c|c|}
\hline Variables & $\beta_{1}$ & $r^{2}$ \\
\hline Ventas- Costos y gastos & $0,7149^{* * *}$ & 0,42 \\
\hline Ventas- Gastos de personal & $0,3212^{* * *}$ & 0,20 \\
\hline
\end{tabular}

Fuente: Elaboración propia.

Significativo a un $0,01^{* * *}$ Software: Stata V.13

Los resultados de la tabla $\mathrm{N}^{\mathrm{0}} 5$ determinan que por cada dólar en que se incrementaron las ventas, los costos y gastos se incrementaron en 0,7149 , y aplicando los mismos criterios para gastos de personal se observa que por cada dólar de aumento en ventas, los gastos de personal aumentaron en 0,32 dólares.

El valor $r^{2}$, también llamado coeficiente de determinación refleja la bondad del ajuste, y cuanto más cerca de 1 se sitúe, mayor será el ajuste del modelo a la variable que se intenta explicar. Se puede observar en la tabla $\mathrm{N}^{0} 5$, que el total de costos y gastos relacionado a las ventas mostró un nivel de ajuste aceptable; y por el contrario, la variable gasto de personal no presentó un nivel adecuado causa - efecto, por lo que se excluyó para el posterior planteamiento del modelo de regresión $\mathrm{ABJ}$.

\section{Modelo de primeras diferencias}

El modelo de primeras diferencias faculta la medición del incremento en la variable dependiente $Y$ ante un incremento en la variable independiente $X$. En el presente estudio, las variables fueron el total de costos y gastos, y ventas respectivamente, de tal manera que en términos monetarios, por cada dólar en que se incrementen las ventas, los costos y gastos se incrementarán en determinado valor. 


$$
C Y G_{t}-C Y G_{t-1}=\beta_{0}+\beta_{1}\left(V_{t}-V_{t-1}\right)+\left(U_{t}-U_{t-1}\right)
$$

Aplicando logaritmos ${ }^{4}$ :

$$
\begin{gathered}
\ln \left(C Y G_{t}-C Y G_{t-1}\right)=\beta_{0}+\beta_{1} \ln \left(V_{t}-V_{t-1}\right)+\left(U_{t}-U_{t-1}\right) \\
\ln \frac{C Y G_{i, t}}{C Y G_{i, t-1}}=\beta_{0}+\beta_{2} \ln \frac{V_{i, t}}{V_{i, t-1}}+U_{i} \\
\ln \Delta C_{i, t}=\beta_{0}+\beta_{1} \ln \Delta V_{i, t}+U_{i, t}
\end{gathered}
$$

Esta última ecuación permite medir la tasa de variación de las ventas y costos y gastos totales ${ }^{5}$, y así aproximarse al desarrollo del modelo ABJ de Anderson.

\section{Modelo ABJ propuesto por Anderson}

Anderson et al. (2003) busca analizar las variaciones cuando las ventas crecen y cuando decrecen, por consiguiente, lo que propone es la adición de una variable dummy, asignando a la variable dummy (dec) el valor de uno cuando las ventas decrecen y cero cuando las ventas crecen, de manera que el modelo final se expresa de la siguiente manera:

$$
\ln \Delta C_{i, t}=\beta_{0}+\beta_{1} \ln \Delta V_{i, t}+\beta_{2} \operatorname{dec} \ln \Delta V_{i, t}+U_{i, t}
$$

El asignar cero a la variable (dec) cuando las ventas crecen, elimina $\beta_{2}$, permitiendo apreciar solo el incremento en las ventas; por el contrario, cuando la variable dec es igual a uno, posibilita que los betas se sumen, mostrando el valor de decremento en las ventas.

El conglomerado de empresas fue clasificado mediante cuartiles, en donde las firmas que representaron un mayor nivel de ventas pertenecen el cuartil número 4, y las de menor nivel de ventas pertenecen al cuartil 1, incluyendo así un indicador de escala de operación basado en el nivel de ventas.

$4 \mathrm{Al}$ adicionar logaritmos a la ecuación, se puede medir le elasticidad de $\mathrm{Y}$ con respecto a $\mathrm{X}$, es decir, se obtiene los cambios porcentuales de $\mathrm{Y}$ con respecto a $\mathrm{X}$, lo que permite medir si existe o no asimetría de costos. (Gujarati y Porter, 2010).

5 Para el cálculo de la tasa de variación de los costos y de las ventas, es necesario determinar el cociente entre el año posterior y el inmediato anterior: $\Delta C Y G_{i, t}=\frac{C Y G_{i, t}}{C Y G A_{i, t-1}}, \Delta V_{i, t}=\frac{V_{i, t}}{V_{i, t-1}}$ 


\section{Tabla 6}

Modelo ABJ de Anderson según cuartiles de ventas

\begin{tabular}{|c|c|c|l|c|c|}
\hline Cuartil & $\boldsymbol{\beta}_{\mathbf{0}}$ & $\boldsymbol{\beta}_{1}$ & \multicolumn{1}{|c|}{$\boldsymbol{\beta}_{\mathbf{2}}$} & Neto & $\boldsymbol{r}^{\mathbf{2}}$ \\
\hline$Q_{1}$ & $-0,4692$ & $0,8643^{* * *}$ & $-0,2423$ & 0,6220 & 0,40 \\
\hline$Q_{2}$ & $-0,3074$ & $0,8841^{* * *}$ & $-0,5515^{* * *}$ & 0,3326 & 0,31 \\
\hline$Q_{3}$ & 0,0528 & $0,8587^{* * *}$ & $-0,0153$ & 0,8434 & 0,64 \\
\hline$Q_{4}$ & 0,1764 & $0,8746^{* * *}$ & $-0,3219^{*}$ & 0,5527 & 0,43 \\
\hline TOTAL & $-0,10799$ & $\mathbf{0 , 8 4 1 3} * * *$ & $-0,193^{* *}$ & $\mathbf{0 , 6 4 8 3}$ & 0,43 \\
\hline
\end{tabular}

Fuente: Elaboración propia.

Significativo a un $* * * 0,01 * * 0,05 * 0,10$. Software: Stata V.13

Prueba F: Hipótesis nula: $\beta 1=\beta 2=0$ Significativo al 0,01

Al analizar los datos en sus valores totales (tabla $\mathrm{N}^{\circ} 6$ ), se puede deducir que por cada $1 \%$ de crecimiento en ventas, los costos crecen en $0,84 \%$; y por cada $1 \%$ de decremento en las ventas, los costos y gastos decrecen en $0,65 \%$, evidenciando un comportamiento asimétrico de los costos en el sector de la construcción en el Ecuador.

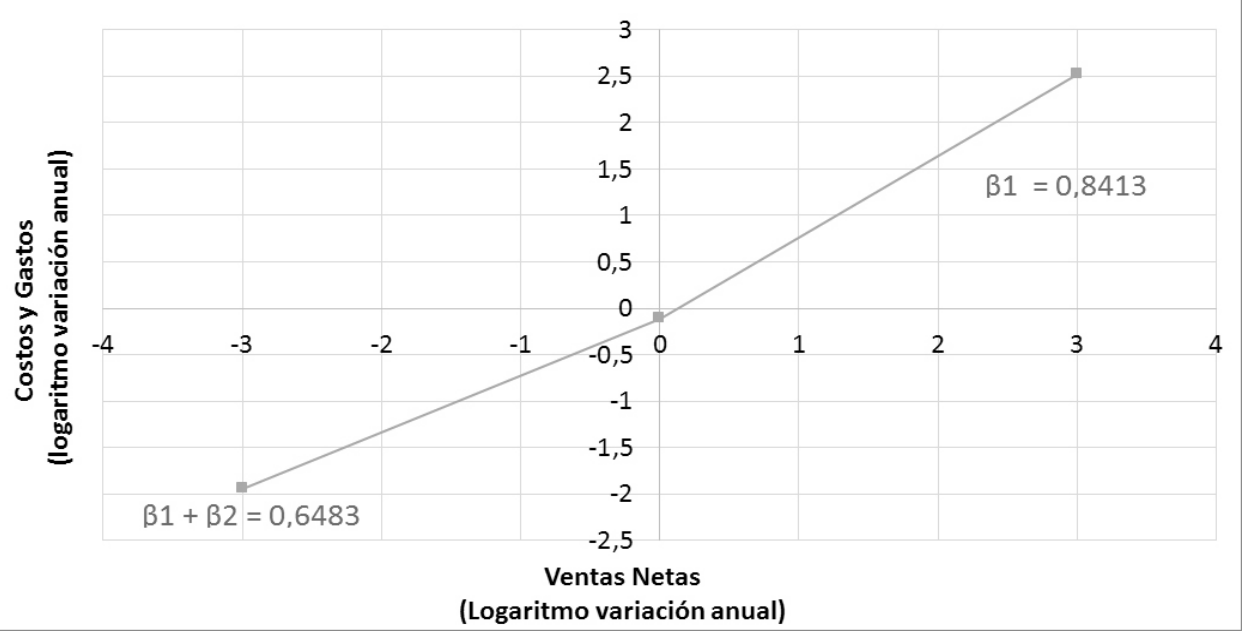

\section{Figura 2}

Regresión de Costos y Gastos modelo ABJ

Fuente: Elaboración propia. 
Como se puede observar en la figura $\mathrm{N}^{\circ} 2$, ante el incremento y decremento de las ventas, los costos varían a diferente velocidad, generando pegajosidad ante los cambios en el nivel de actividad.

Así también, se puede verificar que las empresas del cuartil 2 y 4 presentan un comportamiento asimétrico significativo, con coeficientes $\beta_{2}$ negativos y en valor absoluto menores a $\beta$; es decir, la proporción en que disminuyen los costos es menor que el aumento ante el mismo cambio en el porcentaje de ventas.

Las empresas de cuartil 4, a más de evidenciar significatividad en sus betas, muestran un valor alto en el ajuste $\left(r^{2}=0,43\right)$, es decir, se presentan distintos comportamientos en los costos ante el incremento o disminución de ventas, tomando en cuenta que éste segmento representa una mayor escala de operación.

\section{CONCLUSIONES}

Las empresas ecuatorianas del sector de la construcción analizadas en el presente estudio, muestran un comportamiento asimétrico en el total de costos y gastos, así como también, en gastos de personal.

En el análisis de elasticidades se pudo verificar un comportamiento pegadizo del total de costos y gastos, puesto que ante el incremento de las ventas en $1 \%$, los costos y gastos se incrementaron en $0,94 \%$, mientras que, ante el decremento de ventas en el mismo porcentaje, los costos y gastos se redujeron en $0,30 \%$. De la misma manera, al obtener las variaciones en gastos de personal se puedo observar que ante el incremento del $1 \%$ en las ventas, los gastos se incrementaron en $0,62 \%$, y cuando las ventas disminuyeron en $1 \%$, los gastos de personal lo hicieron en $0,20 \%$, mostrando pegajosidad también en los gastos de personal.

Al aplicar el modelo ABJ de Anderson, se asevera la asimetría de costos, pudiendo observar que ante un incremento de las ventas en $1 \%$ los costos se incrementaron en $0,84 \%$, mientras que, al decremento en la misma proporción, los costos disminuyeron en $0,64 \%$. En este modelo no se consideró los gastos de personal pues el factor de ajuste preliminar no lo amerita. Además, se pudo determinar que en las empresas de mayor escala según el nivel de ventas, el comportamiento asimétrico es mayor. 
La asimetría en el sector puede explicarse por la falta de flexibilidad de las firmas en ajustar los costos y gastos ante los cambios en el nivel de actividad. Los gerentes, ante una reducción en los niveles de ingresos podrían no tomar a tiempo los ajustes necesarios esperanzados en la recuperación de las ventas, manteniendo recursos improductivos que ponen en riesgo la consecución de beneficios.

Los resultados del estudio refuerzan las teorías de la asimetría de costos según el ingreso, y demuestran este comportamiento en el sector de la construcción ecuatoriana, aportando a gerentes e investigadores del área, insumos para el análisis económico y financiero de las organizaciones.

Es pertinente que la administración realice un análisis periódico de las tasas de variación de los costos y gastos en las organizaciones, de forma que constituya un referente más para el análisis financiero, y permita al gerente constructor contar con herramientas para el control y ajuste respectivo en las políticas de manejo económico.

Como futuras líneas de investigación, es necesario un estudio integral de la asimetría de costos en otros sectores de actividad económica, de forma que consolide una metodología de análisis, y permita a la gerencia observar y comparar niveles de asimetría sectoriales.

\section{REFERENCIAS}

1) Anderson, M., Banker, R., y Janakiraman, S. (2003). Are selling, general and administrative costs "sticky"?. Journal of Accountig Research, 41(1), 47-63.

2) Anderson, M., Asdemir, O., y Tripathy, A. (2013). Use of precedent and antecedent information in strategic cost management. Journal of Business Research, 66(5), 643-650.

3) Banker, R., Byzalov, D., y Plehn-dujowich, J. (2014). Demand uncertainty and cost behavior. The Accounting Review, 89(3), 839-865. doi: 10.2308/accr-50661.

4) Cagigal, J., y Petrovic, N. (2017). Comportamiento de los costos en las empresas ecuatorianas. Valor Agregado. Artículo 3, 83-101. doi: 10.2139/ssrn.2997669. 
5) Cooper, R y Kaplan R. (1998). The design of Cost Management Systems: Text, Cases, and Readings ( $2^{\text {nd }}$. Edition). Prentince Hall, Upper Saddle River, NJ.

6) Cunha Marques, A., Silva Santos, C., y Correia Lima, F. (2014). Cost stickiness in latin america open companies from 1997 to 2012. European Scientific Journal, May especial edition ISSN: 1857-7881, 270-281.

7) Gujarati, D. y Porter, D. (2010). Econometría (5ta. Edición). McGrawHill.

8) Horngren, C., Datar, S. y Rajan, M. (2012). Contabilidad de costos. Un enfoque gerencial (14ta edición). Pearson Educación.

9) Instituto Nacional de Estadísticas y Censos INEC. [En línea]. Disponible en: http://www. ecuadorencifras.gob.ec/institucional/home/

10) Malcom, R. (1991). Overhead control implications of activity costing. Accounting Horizons, 69-78.

11) Nassirzadeh, F., Saei, M, Salehi, M., y Bayegi, A. (2013). A study of the stickiness of cost of goods sold an operating costs to changes in sales level in Iran. Studies in Business and Economics, 8(2), 79-89.

12) Norma Internacional de Información Financiera. (2018). Marco conceptual. Recuperado de http://www.ctcp.gov.co/_files/documents/1522857642-8905.pdf

13) Porporato, M., y Werbin, E. (2012). Evidence of sticky costs in banks of Argentina, Brazil y Canada. International Journal of Financial Services Management, 5(4), 303-320. doi: 10.1504/ijfsm.2012.048834.

14) Ribeiro de Medeiros, O y De Souza Costa, P., (2004). Cost stickiness in brazilian firms. Social Science Research Network. doi: 10.2139/ssrn.632365.

15) Stimolo, M. (2016). Análisis del Comportamiento Pegadizo de los costos en Empresas Argentinas que cotizan en el mercado. (Doctorado). Universidad Nacional de Córdoba. Recuperado de https://rdu.unc.edu.ar/bitstream/handle/

16) Superintendencia de Compañías, Valores y Seguros del Ecuador. [En línea]. Disponible en: http://appscvs.supercias.gob.ec/portalInformacion/sector_societario.zul

17) West, D. (2003). Three financial strategies. Journal of Health Care Finance, 30(1), 10-22. 